\title{
Rectal bleeding in adults over 20 years: Endoscopic investigations and results in current hospital practices in Yaoundé, Cameroon
}

\author{
Firmin Ankouane Andoulo ${ }^{1}$, Dominique Noah Noah ${ }^{2 *}$, Roger Djapa ${ }^{1}$, Michele Tagni Sartre ${ }^{3}$, \\ Elie Claude Ndjitoyap Ndam $^{4}$, Kathleen Ngu Blacket ${ }^{1}$ \\ ${ }^{1} \mathrm{CHU}$ Yaounde, Department of Internal Medicine and Specialties, Faculty of Medicine and Biomedical Sciences, University of \\ Yaounde I, Yaounde, Cameroon \\ ${ }^{2}$ Yaounde Central Hospital, Department of Internal Medicine, Faculty of Medicine and Pharmaceuticals Sciences, University of \\ Douala, Douala, Cameroon \\ ${ }^{3}$ La Cathédrale Medical Centre, Yaounde, Cameroon \\ ${ }^{4}$ Yaounde General Hospital, Department of Internal Medicine and Specialties, Faculty of Medicine and Biomedical Sciences, Uni- \\ versity of Yaounde I, Yaounde, Cameroon \\ Email: noahnoahd@yahoo.fr
}

Received 25 July 2013; revised 29 August 2013; accepted 12 September 2013

Copyright (C) 2013 Firmin Ankouane Andoulo et al. This is an open access article distributed under the Creative Commons Attribution License, which permits unrestricted use, distribution, and reproduction in any medium, provided the original work is properly cited.

\begin{abstract}
Introduction: In order to identify the modes of investigation and the results of the assessment of rectal bleeding in the Cameroonian adult, we retrospectively analysed the records of 287 patients aged above 20 years diagnosed with rectal bleeding with the aim to know the prescription patterns according to age, the diagnostic performance of tests and the results. Methodology: Between the $1^{\text {st }}$ of January 2009 and the $30^{\text {th }}$ of June 2010, we examined patients at the University Teaching Hospital and the "La Cathédrale" Medical Centre in Yaounde. Age, sex, endoscopic tests and results were evaluated. Results: 287 protocols met our selection criteria, sex ratio (M/F) 2.4/1, median age 46 years interquartile range $[36,55]$. Normal tests were 57 (19.2\%). Main lesions: haemorrhoids (42.4\% CI95 36.7 - 48.3), colorectal cancer (10.8\% CI95 7.5 - 14.9), anal fissure (8.8\% CI95 5.8 - 12.6) and colorectal polyps (8.4\% CI95 5.5 - 12.2). The prevalence of significant lesions (polyps and cancer) recorded $7 \%$ for those under age $40.20 .5 \%$ in those were between 40 - 50 years, and $28.9 \%$ for those over 50 years. For anoscopies, $\mathbf{4 4 . 4 \%}$ were under 40 years, $39.3 \%$ of cases of sigmoidoscopy affected those between 40 - 50 years and colonoscopy affected $54 \%$ of those over 50 years. For the diagnostic yield, 26.2\% had a significant lesion for flexible sigmoidoscopy and only $\mathbf{1 6 . 7 \%}$ and $\mathbf{1 . 6 \%}$ for colonoscopy and anoscopy
\end{abstract}

${ }^{*}$ Corresponding author. respectively. Conclusion: The study shows that anoscopy and sigmoidoscopy are the main initial tests conducted in Cameroon in the assessment of rectal bleeding in adults of less than $\mathbf{5 0}$ years and they are quite sufficient. Haemorrhoids and colorectal cancer are the main pathologies.

Keywords: Rectal Bleeding; Colon Polyps; Colorectal Cancer; Sigmoidoscopy; Colonoscopy; Cameroon Cancer

\section{INTRODUCTION}

Rectal bleeding accounts for $20 \%$ of gastrointestinal bleeding. It is equal to the most common detection mode for lower gastrointestinal bleeding especially in the elderly [1]. Several studies have been conducted in order to know what mode of investigation is appropriate depending on the age of the patients, the risk of colorectal cancer and the clinical presentation mode [2-4]. Even though colonoscopy is unanimously recommended for patients aged 50 and above in the assessment of rectal bleeding [5], there seem to be no consensus guidelines on how to deal with rectal bleeding in young patients under the age of $50[1,3,6,7]$.

In sub-Saharan Africa and more specifically Cameroon, few studies have focused on the topic and there is no consensus $[1,8,9]$.

We sought to know, through a retrospective study, which investigation modes existed in our context and the 
results found while reviewing cases of the rectal bleeding following the patients' ages, especially in the diagnosis of significant lesions such as colorectal polyps and colorectal cancer.

\section{MATERIALS AND METHODS}

We conducted a retrospective study on the medical history of all patients aged 20 and above who had carried out an endoscopic assessment for rectal bleeding in Yaounde, Cameroon, from $1^{\text {st }}$ January 2009 to $30^{\text {th }}$ June 2010. These were patients of the University Teaching Hospital (CHU) Yaounde and La Cathédrale Medical Centre Yaoundé (CMC). Investigative tests used were anoscopy, flexible sigmoidoscopy and colonoscopy.

The tests which were excluded were colonoscopies with poor preparation, sigmoidoscopies with poor preparation, protocols indicating the diagnosis of a rectal tumor palpable on rectal examination, the examination of patients below 20 years and incomplete file protocols for the analysis of variables. Only one test was administered per patient during the study.

Variables recorded were as follows: demographic characteristics (age, gender), the types of tests required (anoscopy, flexible sigmoidoscopy, colonoscopy) and test results (normal or presence of a colorectal lesion).

Data was entered and analyzed using the French version of Epi Info 6.04 software and Excel 2007. For quantitative variables, the means, standard deviations, medians and interquartile ranges (IQR) were calculated. Proportions were set for qualitative variables together with their confidence intervals at $95 \%(95 \% \mathrm{CI})$.

To examine the relationship between two discrete variables, we used the Pearson's $\chi^{2}$ test using the Yates' correction and Fisher's exact test for reduced samples, for an accepted significance level of $5 \%$.

\section{RESULTS}

\subsection{Sample Population (Table 1)}

During the study period, a total of 312 protocols for endoscopic tests carried out in adult patients in rectal bleeding records were analysed. Two hundred and ninetyseven $(95.2 \%)$ protocols sampled met our inclusion criteria. Of all the patients, $100(33.7 \%)$ were under 40 years, $83(27.9 \%)$ were between 40 and 50 years and 114 $(38.4 \%)$ were above 50 years of age. In terms of gender, there were $209(70.4 \%)$ men and 88 (29.6\%) women with an $\mathrm{M} / \mathrm{F}$ sex ratio of $2: 4$. The average age was 46 years IQR [36 years, 55 years] and the modal age was 55 years.

As for the tests conducted, $124(41.7 \%)$ tests were anoscopies, $84(28.3 \%)$ were flexible sigmoidoscopies and $89(30 \%)$ took colonoscopy tests. Of these tests, 55 (44.4\%) anoscopies concerned patients under 40 years,
$33(39.3 \%)$ flexible sigmoidoscopies involved patients aged between 40 - 50 years and 48 (54\%) colonoscopies were done on patients over 50 years.

\subsection{Results of Endoscopies in the Assessment of Rectal Bleeding in the Cameroonian Adult (Table 2)}

The results were normal in $57(19.2 \%)$ tests. The predominant lesions were haemorrhoids with 126 cases (42.4\%, 95\% CI: 36.7 to 48.3$)$, followed by colorectal cancer, 32 cases $(10.8 \%, 95 \%$ CI 7.5 to 14,9$)$, anal fissures recorded 26 cases $(8.8 \%, 95 \%$ CI 5.8 to 12.6$)$ and colorectal polyps 25 cases $(8.4 \%, 95 \%$ CI 5.5 to 12.2$)$. Exceptionally, colitis recorded 12 cases $(4.0 \%, 95 \%$ CI 2.1 to 7.0$)$, colonic diverticula 11 cases $(3.7 \%, 95 \% \mathrm{CI}$ 1.1 to 6.5$)$, rectal ulcers 6 cases $(2 \%, 95 \% \mathrm{CI}: 0.7-4.3)$ and rectal varices 1 case $(0.3 \%, 95 \% \mathrm{CI}: 0.0-1.9)$.

\subsection{The Prevalence of Significant Lesions According to the Ages of Patients with Rectal Bleeding (Table 3)}

In patients below 40 years, $7(7 \%)$ significant lesions were found (including $3(3 \%)$ colorectal cancers and 4

Table 1. Distribution of tests according to age groups in the assessment of rectal bleeding in Cameroonian adults.

\begin{tabular}{ccccc}
\hline & $\begin{array}{c}\text { Anoscopies } \\
(\mathrm{n}=124)\end{array}$ & $\begin{array}{c}\text { Sigmoidoscopy } \\
(\mathrm{n}=84)\end{array}$ & $\begin{array}{c}\text { Colonoscopies } \\
(\mathrm{n}=89)\end{array}$ & $\begin{array}{c}\text { Total } \\
(\mathrm{n}=297)\end{array}$ \\
\hline \multicolumn{5}{c}{ Workforce by age, $n(\%)$} \\
$<40$ years old & $100(33.7)$ & $55(44.4)$ & $22(26.2)$ & $23(25.8)$ \\
$40-50$ years & $83(27.9)$ & $32(25.8)$ & $33(39.3)$ & $18(20.2)$ \\
$>50$ years & $114(38.4)$ & $37(29.8)$ & $29(34.5)$ & $48(54)$ \\
\hline
\end{tabular}

Table 2. Prevalence of colorectal lesions found in reports on rectal bleeding by endoscopy in Cameroonian adults $(n=297)$.

\begin{tabular}{cccc}
\hline Results & Employees & (Percentage) & $\begin{array}{c}\text { Confidence } \\
\text { interval 95\% }\end{array}$ \\
\hline Angiodysplasia & 1 & 0.3 & $0.0-1.9$ \\
Cancer & 32 & 10.8 & 7.5 to 14.9 \\
Colitis & 12 & 4.0 & 2.1 to 7.0 \\
Diverticulum & 11 & 3.7 & 1.1 to 6.5 \\
Anal fissure & 26 & 8.8 & 5.8 to 12.6 \\
Haemorrhoids & 126 & 42.4 & 36.7 to 48.3 \\
Colonic polyps & 25 & 8.4 & 5.5 to 12.2 \\
Solitary rectal ulcer & 6 & 2.0 & $0.7-4.3$ \\
Rectal varices & 1 & 0.3 & $0.0-1.9$ \\
Normal & 57 & 19.2 & $14.9-24.1$ \\
\hline
\end{tabular}


polyps (4\%) colorectal polyps) in patients aged between 40 - 50 years, $17(20.5 \%)$ significant lesions were found (including $10(12.0 \%)$ colorectal cancers and $7(8.4 \%)$ colorectal polyps) and in patients over 50 years, 33 (28.9\%) significant lesions were found (including 19 $(16.7 \%)$ colorectal cancers and $14(12.3 \%)$ colorectal polyps). In the univariate analysis, significant lesions commoner in patients aged above 50 years (Relative Risk: $2.21,95 \%$ CI: 1.38 to $3.53, p=0.0007)$.

\subsection{Contribution of Endoscopy in the Diagnosis of Significant Lesions in Adult Patients with Rectal Bleeding (Table 4)}

Significant lesions were detected in $33(16.7 \%)$ patients (17 (19.1\%) colorectal cancers and $16(17.8 \%)$ colorectal polyps) with rectal bleeding using colonoscopy. Flexible sigmoidoscopy helped in detecting significant lesions in $22(26.2 \%)$ patients (including $14(16.7 \%)$ colorectal cancers and $8(9.5 \%)$ colorectal polyps). Finally, significant lesions were detected by anoscopy in $2(1.6 \%)$ patients (including $1(0.8 \%)$ colorectal cancers and 01 $(0.8 \%)$ colorectal polyp). In the univariate analysis, the diagnosis of significant lesions was strongly connected to colonoscopy compared with other endoscopic tests (Relative Risk: $3.21,95 \%$ CI 2.02 to 5.11$), \mathrm{p}<10^{-5}$ ).

\section{DISCUSSION}

Colonoscopy is considered the best endoscopic method in assessing and managing rectal bleeding $[4,10,11]$. Today, there are no consensus guidelines on its formal in-

Table 3. Prevalence of significant lesions according to patients' age groups reports on rectal lesions in the Cameroonian adult.

\begin{tabular}{lccc}
\hline & \multicolumn{3}{c}{ Age group } \\
\hline & $\begin{array}{c}<40 \text { years } \\
(\mathrm{n}=100)\end{array}$ & $\begin{array}{c}40-50 \text { years } \\
(\mathrm{n}=83)\end{array}$ & $\begin{array}{c}>50 \text { years } \\
(\mathrm{n}=114)\end{array}$ \\
\hline Significant lesions, $\mathrm{n}(\%)$ & $7(7)$ & $17(20.5)$ & $33(28.9)$ \\
Colorectal cancer, $\mathrm{n}(\%)$ & $3(3)$ & $10(12.0)$ & $19(16.7)$ \\
Colorectal polyps, $\mathrm{n}(\%)$ & $4(4)$ & $7(8.4)$ & $14(12.3)$ \\
\hline
\end{tabular}

Table 4. Prevalence of significant lesions according to endoscopy in the assessment of rectal lesions in the Cameroonian adult.

Endoscopic tests

\begin{tabular}{lccc}
\hline & Anoscopies & Sigmoidoscopy & Colonoscopies \\
$(\mathrm{n}=124)$ & $(\mathrm{n}=84)$ & $(\mathrm{n}=89)$ \\
\hline Significant lesions, $\mathrm{n}(\%)$ & $2(1.6)$ & $22(26.2)$ & $33(37.1)$ \\
Colorectal cancer, n (\%) & $1(0.8)$ & $14(16.7)$ & $17(19.1)$ \\
Colorectal polyps, n (\%) & $1(0.8)$ & $8(9.5)$ & $16(17.8)$ \\
\hline
\end{tabular}

dication in patients under 40 years who suffer from rectal bleeding [3,6,10]. Bhargava et al. [12] further argued that the predominant lesions in rectal bleeding cases in tropical countries and the tests conducted were different from those reported in Western countries. The study's aim was to analyse test prescription practices in the process of diagnosing rectal bleeding in the Cameroonian adult, taking into account age, and to report the results of these study, and most especially, significant lesions. We observed that colonoscopy was the second review after Anoscopy, prescribed in our community, especially in patients over 50 years. In patients less than 40 years, anoscopy was the first test used to investigate rectal bleeding and in patients between 40 - 50 years, flexible sigmoidoscopy was the first-line examination. Haemorrhoids $(42.4 \%)$, colorectal cancer $(10.8 \%)$, anal fissure $(8.8 \%)$ and colorectal polyps $(8.4 \%)$ were the predominant lesions. Significant lesions, particularly colorectal cancer were common in patients above 50 years.

The specificity of rectal bleeding in our country is twofold. First, it is assumed in our context that haemorrhoidal disease cannot be treated by way of modern me dicine, but only through traditional medicine. Also, when people suspect that they may be suffering from amoebic colitis caused by rectal bleeding they resort to self-medication using metronidazole and this is common practice among the population. This explains why patients arrive too late at the hospital and in fewer numbers. This peculiarity may have had an impact on our results, especially in the prevalence of colorectal cancer. The alarm symptoms that we did not identify in this work could be the reasons that led our patients to consult. This is a retrospective study and we could have excluded patients with alarm symptoms so as to avoid selection bias. However, the study as far as we know is the first in our country. It provides an overview of the medical prescriptions in case of rectal bleeding. The legal framework for prescribing examinations is not observed in Cameroon. Thus, the traditional practitioner, paramedics, general practitioners and other specialists besides gastroenterologist may prescribe an investigation test.

The incidence of rectal bleeding increases with age and corresponds with an increasing frequency of gastrointestinal diseases and this is specific to each region. In our study, it was observed that patients above 50 years (38.4\%) and those under 40 years $(33.7 \%)$ were the most affected. We did not find any justification for this. On the contrary, comorbidity and some medications such as NSAIDs and anticoagulants are known to account for the lower gastrointestinal bleeding in the elderly. The main lesions found in our study were different, apart from haemorrhoids, from those reported in some tropical countries $[8,9,12]$. Rather, they were similar to those reported by Mbengué et al. [1] in Dakar, Senegal. Djibril et al. [8] 
in Lome, Togo limited their study to subjects aged between 24 - 45 years; individuals aged 50 and above were excluded. These subjects are known to be most affected by colorectal cancer [13] cancer which could explain the low prevalence $(5.88 \%)$ of colorectal cancer in this series compared to ours. Also, Assi et al. [9] in Abidjan, Côte d'Ivoire, reported a prevalence of colorectal cancer of $5.53 \%$ among adult subjects with rectal bleeding using colonoscopy as a diagnostic test. The difference compared to our series would be based on the type of diagnostic tests. It is important to note the high prevalence of colorectal cancer in our study and in that of Mbengué et al. [1] of $10.8 \%$ and $11.9 \%$ respectively. Changes in our eating habits have been observed in recent years, initially predominantly vegetarian and meat-rich today may partly explain these results.

The prevalence of significant lesions in patients 40 50 years and in patients over 50 years was high, whereas in patients below 40 years, it was low. These results are consistent with those contained in the literature and especially those in the work of Lewis et al. [6] who reported similar results in a series of patients under 50 years with rectal bleeding. During the study and that of Lewis et al. [6] the warning signs which are changing bowel habits, iron deficiency anaemia and weight loss, these signs are known to have a high positive predictive value [14-16] in diagnosing colorectal cancer in a patient with signs of rectal bleeding which were not mentioned.

The diverticular bleeding is a common cause of rectal bleeding in the West [17]. In sub-Saharan Africa, diverticular disease is a rare disease [18] and diverticular bleeding is rarely described [19]. The $3.7 \%$ prevalence rate is therefore in accordance with these arguments. Bleeding from rectal ulcer seemed relatively frequent compared to the literature [20,21]. Lin et al. [21] have identified comorbid conditions associated with this disease including diabetes, bed-rest, renal failure, hypoglycaemia albumin, cerebrovascular accidents, respiratory failure and atherosclerosis. None of these factors was reported in our series. Their cause is not mechanical.

The diagnostic value for significant lesions, particularly colorectal cancer was higher with flexible sigmoidoscopy $(26.2 \%)$ for lesions of the distal segment of the colon. This is not a new observation [11,22,23]. We noted that $65.5 \%$ of flexible sigmoidoscopy was performed in patients less than 50 years. This test is considered sufficient in the assessment of rectal bleeding without alarming symptoms in patients less than 50 years by some authors which is due to the preferential localization of significant lesions in the distal colon in this age group on the one hand $[2-4,11,24]$, but also due to a low prevalence of significant lesions, particularly colorectal cancer in this age group $[6,16,25,26]$. Marderstein et al. [10] in a study of simple rectal bleeding emphasised that the diagnostic yield of colonoscopy was low in indicating this. The authors added that flexible sigmoidoscopy was sufficient to exclude significant injury if other formal indicators for colonoscopy were not associated. We drew three arguments from the findings in relation to our study: 1) that flexible sigmoidoscopy was sufficient in the assessment of our patients under 50 years, 2) that the performance of this test was high and 3) that our patients probably had warning signs associated with rectal bleeding to explain the high frequency of significant lesions in the age group of those below 50 years $(27.5 \%)$. Late arrival to the hospital as already mentioned is a contributing factor.

Colonoscopy in the assessment of rectal bleeding without warning signs has a low yield according to some authors $[10,14,23]$ and is costly for a patient who has no health insurance. In Cameroon, the price of a colonoscopy is three times that of sigmoidoscopy. Colonoscopy was the second examination prescribed in our study $(30 \%)$, especially in patients over 50 years. It is recommended by most published guidelines and EPAGE II criteria (available at http://www.epage.ch) in screening for colorectal cancer in patients at high risk of colorectal cancer [27] and in subjects aged above 50 years. Risk factors for colorectal cancer and alarm symptoms were not identified in this study. However, the contribution of colonoscopy was instrumental in the diagnosis of significant lesions. Late arrivals at hospitals explain this result.

\section{CONCLUSION}

The absence of a legal framework and a consensus on the follow-up of rectal bleeding on the one hand and the traditional methods of individuals in the face of rectal bleeding on the other hand are major obstacles in assisting the patient. However, the requirements of investigative tests do not really deviate from what is recommended in several publications in the existing literature. Test results need to be reported taking into account the characteristics of rectal bleeding and its associated warning signs. The high cost of testing and the lack of health coverage oblige one to adopt a systematic approach focusing on anoscopy and sigmoidoscopy such as initial tests for young patients with no warning signs and colonoscopy for elderly patients or those at the risk of colorectal cancer.

\section{REFERENCES}

[1] Mbengue, M., Dia, D., Diouf, M.L., Bassène, M.L., Fall, S., Diallo, S., et al. (2009) Apport de la coloscopie dans le diagnostic des rectorragies à Dakar (Sénégal). Médecine Tropicale, 69, 286-288.

[2] Nikpour, S. and Ali Asgari, A. (2008) Colonoscopic evaluation of minimal rectal bleeding in average-risk patients 
for colorectal cancer. World Journal of Gastroenterology, 14, 6536-6540. http://dx.doi.org/10.3748/wjg.14.6536

[3] Khalid, A.B., Majid, S., Salih, M. and Jafri, W. (2011) Is full colonoscopic examination necessary in young patients with fresh bleeding per rectum? Endoscopy, 43, 692-696. http://dx.doi.org/10.1055/s-0030-1256356

[4] Wong, R.F., Khosla, R., Moore, J.H. and Kuwada, S.K. (2004) Consider colonoscopy for young patients with hematochezia. Journal of Family Practice, 53, 879-884.

[5] Peytremann-Bridevaux, I., Arditi, C., Froehlich, F., O'Malley, J., Fairclough, P., Le Moine, O., et al. (2009) Appropriateness of colonoscopy in Europe (EPAGE II). Irondeficiency anaemia and hematochezia. Endoscopy, 41, 227-233. http://dx.doi.org/10.1055/s-0028-1119644

[6] Lewis, J.D., Shih, C.E. and Blecker, D. (2001) Endoscopy for hematochezia in patients under 50 years of age. Digestive Diseases and Sciences, 12, 2660-2665. http://dx.doi.org/10.1023/A:1012715110440

[7] Burling, D., East, J.E. and Taylor, S.A. (2007) Investigating rectal bleeding. $B M J, 335,1260-1262$. http://dx.doi.org/10.1136/bmj.39379.598090.AD

[8] Djibril, A.M., M’ba, K.B., Bagny, A., Kaaga, L., Redah, D. and Agbetra, A. (2009) Etiologies des rectorragies chez l'adulte en milieu Africain: A propos de 85 cas colligés en 12 ans au Chu-Campus de Lomé. Mali Médical, 24, 40-42.

[9] Assi, C., Lohoues-Kouacou, M.J., Toth'o, A., Traoré, F., Allah-Kouadio, E. and Camara, B.M. (2006) Quelques aspects épidémiologiques des rectorragies chez l'adulte noir Africain. Médecine d'Afrique Noire, 5, 315-319.

[10] Marderstein, E.L. and Church, J.M. (2008) Classic outlet rectal bleeding does not require full colonoscopy to exclude significant pathology. Diseases of the Colon \& Rectum, 2, 202-206.

http://dx.doi.org/10.1007/s10350-007-9123-1

[11] Choi, H.K., Law, W.L. and Chu, K.W. (2003) The value of flexible sigmoidoscopy for patients with bright red rectal bleeding. Hong Kong Medical Journal, 9, 171-174.

[12] Bhargava, D.K., Rai, R.R. and Chopra, P. (1990) Colonoscopy for investigation of unexplained rectal bleeding in tropical country. Journal of Gastroenterology, 6, 781785.

[13] Karsten, B., Kim, J., King, J. and Kumar, R.R. (2008) Characteristics of colorectal cancer in young patients at an urban county hospital. American Surgeon, 74, 973976.

[14] Adelstein, B.A., Macaskill, P., Chan, S.F., Katelaris, P.H. and Irwig, L. (2011) Most bowel cancer symptoms do not indicate colorectal cancer and polyps: A systematic review. BMC Gastroenterology, 30, 65 . http://dx.doi.org/10.1186/1471-230X-11-65

[15] Astin, M., Griffin, T., Neal, R.D., Rose, P. and Hamilton, W. (2011) The diagnostic value of symptoms for colorectal cancer in primary care: A systematic review. British Journal of General Practice, 61, 231-243. http://dx.doi.org/10.3399/bjgp11X572427
[16] Robertson, R., Campbell, C., Weller, D.P., Elton, R., Mant, D., Primrose, J., et al. (2006) Predicting colorectal cancer risk in patients with rectal bleeding. British Journal of General Practice, 56, 763-767.

[17] Wilkins, T., Baird, C., Pearson, A.N. and Schade, R.R. (2009) Diverticular bleeding. American Family Physician, 80, 977-983.

[18] Mbengué, M., Dia, D. and Diouf, M.I. (2009) La diverticulose colique à Dakar: Aspects épidémiologiques, cliniques et endoscopiques chez 34 patients. Journal Africain d'Hépato-Gastroentérologie, 3, 112-114.

[19] Ankouane Andoulo, F., Tagni-Sartre, M., Dang Babagna, I. and Ndjitoyap Ndam, E.C. (2012) Hémorragie diverticulaire colique rare chez un noir africain: A propos d'un cas. Journal Africain d'Hépato-Gastroentérologie, 6, 6871.

[20] Motomura, Y., Akahoshi, K., Matsui, N., Kubokawa, M., Higuchi, N., Oda, M., et al. (2010) Clinical and endoscopic characteristics of acute hemorrhagic rectal ulcer, and endoscopic hemostatic treatment: A retrospective study of 95 patients. Colorectal Disease, 12, e320-e325. http://dx.doi.org/10.1111/j.1463-1318.2009.02091.x

[21] Lin, C.K., Liang, C.C., Chang, H.T., Hung, F.M. and Lee, T.H. (2011) Acute hemorrhagic rectal ulcer: An important cause of lower gastrointestinal bleeding in the critically ill patients. Digestive Diseases and Sciences, 56 3631-3637. http://dx.doi.org/10.1007/s10620-011-1759-y

[22] Hafeez Bhatt, A.B. and Quraishy, M.S. (2011) Flexible sigmoidoscopic findings in patients with fresh rectal bleeding. Journal of College of Physicians and Surgeons Pakistan, 21, 577-578.

[23] Thompson, M.R., Flashman, K.G., Wooldrage, K., Rogers, P.A., Senapati, A., O'Leary, D.P., et al. (2008) Flexible sigmoidoscopy and whole colonic imaging in the diagnosis of cancer in patients with colorectal symptoms. British Journal of Surgery, 95, 1140-1146. http://dx.doi.org/10.1002/bjs.6234

[24] Kumar, R.R., King, J., Holt, A., Huynh, R., Mittal, R., Deen, R., et al. (2007) Prevalence of left-sided colorectal cancer and benefit of flexible sigmoidoscopy: A county hospital experience. American Surgeon, 73, 994-997.

[25] Zbuk, K., Sidebotham, E.L., Bleyer, A. and La Quaglia, M.P. (2009) Colorectal cancer in young adults. Seminars in Oncology, 36, 439-450. http://dx.doi.org/10.1053/j.seminoncol.2009.07.008

[26] Mathew, J., Shankar, P. and Aldean, I.M. (2004) Audit on flexible sigmoidoscopy for rectal bleeding in a district general hospital: Are we over-loading the resources? Postgraduate Medical Journal, 80, 38-40. http://dx.doi.org/10.1136/pmj.2003.008284

[27] Arditi, C., Peytremann-Bridevaux, I., Burnand, B., Eckardt, V.F., Bytzer, P., Agréus, L., et al. (2009) Appropriateness of colonoscopy in Europe (EPAGE II). Screening for colorectal cancer. Endoscopy, 41, 200-208. http://dx.doi.org/10.1055/s-0028-1119626 